\title{
Posture-Invariant Statistical Shape Analysis Using Laplace Operator
}

\author{
Stefanie Wuhrer ${ }^{\mathrm{b}, \mathrm{c}}$, Chang Shu ${ }^{\mathrm{a}}$, Pengcheng $\mathrm{Xi}^{\mathrm{a}}$ \\ ${ }^{a}$ National Research Council of Canada \\ ${ }^{b}$ Saarland University, Germany \\ ${ }^{c}$ Max Plank Institute Informatik, Germany
}

\begin{abstract}
Statistical shape analysis is a tool that allows to quantify the shape variability of a population of shapes. Traditional tools to perform statistical shape analysis compute variations that reflect both shape and posture changes simultaneously. In many applications, such as ergonomic design applications, we are only interested in shape variations. With traditional tools, it is not straightforward to separate shape and posture variations. To overcome this problem, we propose an approach to perform statistical shape analysis in a posture-invariant way. The approach is based on a local representation that is obtained using the Laplace operator.
\end{abstract}

\section{Introduction}

Statistical shape analysis is a tool that allows to quantify the shape variability of a population of shapes. This tool helps to gain a thorough understanding of a population of shapes, and it has various applications, such as designing products for a specific target population, computing shape priors of a population to aid in solving statistical inference problems, and comparing different populations.

For applications that require a user to understand certain properties of a population, it is helpful to visualize the shape variations of the population. An example of such an application is the design of garments or gear for a target population of human subjects.

In most ergonomic design applications, we are interested in computing the shape variations of a database of shapes in a standard posture. Anthropometric surveys are conducted to aid the study of shape variations of a population sample. However, even when subjects are instructed to maintain a standard posture, there are slight posture variations, especially in the position of the arms. For example, this occurs in the Civilian American and European Surface Anthropometry Resource (CAESAR) database [1].

In some ergonomic design applications, it is important for the product to fit a typical subject of the population even when the subject moves. Hence, for this application, we are interested in computing the shape variations of a database of shapes captured in varying postures.

A popular approach to perform shape analysis is to represent the shapes using their vertex coordinates and to analyze these shapes using approaches such as principal component analysis. This approach has the advantage that reconstructing a shape based on its vertex coordinate representation is straightforward, which allows an intuitive visualization of the shape variability. However, for a population of shapes in different postures, approaches operating on vertex coordinates compute variations that reflect both shape and posture changes of the models simultaneously. It is not straightforward to extract only shape variations using these approaches.

To overcome this problem, we propose a method to perform statistical shape analysis of a population of shapes in varying postures in a posture-invariant way, and to visualize the resulting shape variations in a standard posture. To achieve this, we develop a posture-invariant shape representation based on the Laplace operator, and we perform statistical shape analysis using this representation. Since we aim to visualize the shape variability of a population, this shape representation needs to allow for the reconstruction of a shape based on its representation. We will show that computing the representation from a given model is straightforward. Furthermore, we will present a solution to the more challenging problem of computing a model from the representation.

\section{Related Work}

To perform statistical analysis on a population of shapes, we require intrinsic correspondence information between the shapes. Much work has been conducted to compute correspondences between pairs and populations of shapes, see [2] for a recent survey on the topic.

Once the database of models is in correspondence, corresponding vertices on different models have the same index. This correspondence information is 
then commonly used to find the best rigid alignment of the shapes using generalized Procrustes analysis [3]. After this step, a common representation is to model each shape using a vector of vertex coordinates $\left[x_{1}, y_{1}, z_{1}, \ldots, x_{n}, y_{n}, z_{n}\right]^{T}$, where $x_{i}\left(y_{i}, z_{i}\right.$, respectively) is the $x$ - $(y-, z-$, respectively) coordinate of the vertex with index $i$. In the following, we review a few statistical shape analysis tools that are commonly used in combination with this shape representation.

Principal Component Analysis (PCA) is a standard tool to perform linear shape analysis of a set of shapes represented by vectors in a Euclidean vector space. An average object is computed as the linear average of the data set. PCA then finds the linear $d$-dimensional subspace of the vector space that maximizes the variability of the model. Here, $d$ is a positive integer smaller than the input dimension. PCA has been applied to compute the shape variation of a population of human shapes in a standard posture [4]. While this approach yields satisfactory results when computing the shape variation of models in a standard posture, it is not suitable for computing the shape variation of models in varying postures. The reason is that in this case, some of the principal components reflect posture changes of the models.

To allow for posture changes, Fletcher et al. [5] propose an approach called Principal geodesic analysis. This approach aims to simulate PCA in a Riemannian symmetric space. By using this space rather than $\mathrm{Eu}-$ clidean space, posture variations are less strongly emphasized. While the approach is general, one needs to be able to efficiently compute geodesics to use this method. The original method by Fletcher et al. therefore focuses on shapes represented by a medial axis description.

Nain et al. [6] aim to perform localized statistical shape analysis. That is, rather than looking at global shape variations, they are interested in localized shape changes on the surface. To compute these localized shape statistics, they employ spherical wavelets. While this tool allows for localized statistical shape analysis, it cannot directly be used to overcome the problems caused by posture changes.

Wang et al. [7] propose an approach called multivariate tensor-based morphometry. This approach maps each surface to a canonical representation called a holomorphic form and computes a parameterization in this canonical space. The method then performs statistics based on the deformation in the canonical space. This approach therefore emphasizes deformations of the models and marks them as unusual. In this paper, we aim to perform statistics on the shape only for a set of models given in varying postures.

Vasilescu and Terzopoulos [8] propose a nonlinear, multifactor model that generalizes Independent Component Analysis (ICA). The multilinear ICA learns the statistically independent components of multiple factors related to two-dimensional images. They apply the multilinear analysis to sets of facial images that combine different facial geometries, expressions, head poses, and lighting conditions. Allen et al. [4], Anguelov et al. [9], and Hasler et al. [10] propose statistical models to encode human body shape. Allen et al. only encode the shape variation in a standard posture. Anguelov et al. treat human body pose and human body shape independently, and perform PCA on each space. Hasler et al. propose a representation that jointly encodes both human body shape and pose and that is locally translation and rotation invariant. By using this model to perform statistical analysis, human body shape and pose are treated as correlated entities. While these methods allow to analyze shape and posture, they cannot be used to compute shape variations from a population of shapes in varying postures.

For human bodies, much research is focused on estimating the pose, i.e. the articulated skeletal structure, of the human body from partial information, such as a single image [11]. While for human populations, an estimate of the pose could be used to differentiate between shape and posture, our approach operates on the surface directly without the need for an estimated pose of the subject.

We propose to use a method based on the Laplace operator to perform posture-invariant statistical shape analysis. The Laplace operator has previously been used for mesh smoothing and regularization [12], for surface editing [13], and for 3D mesh fitting [14]. Furthermore, the spectrum of the Laplace operator has been used for mesh processing in a similar way to the Fourier transforms of images [15], and for shape matching and dissimilarity computation of volumetric data [16] and of triangle meshes [17]. Baran et al. [18] used differential coordinates over local connected patches of a mesh to define a shape representation that is used to transfer mesh deformations from one character to another one while preserving the semantic meaning.

Our method uses local frames to derive a postureinvariant representation of a surface. Yu et al. [19] used a similar representation for mesh editing. Their framework allows both local and global mesh editing. The method uses a mesh solver based on the Poisson equation. Local frames are used to represent boundary conditions since this allows to encode the difference between an undeformed and a deformed frame uniquely using a rotation and a scale factor.

\section{Approach}

We propose an approach to perform postureinvariant shape analysis. The input to our algorithm is a database $S_{1}, S_{2}, \ldots, S_{m}$ of possibly incomplete 
3D models. We start by parameterizing the shapes using a template-based approach to obtain a set of 3D shapes $X_{1}, X_{2}, \ldots, X_{m}$ represented by triangular manifold meshes with the same connectivity.

The approach then proceeds in three steps. First, the meshes are represented in a posture-invariant way. Second, shape analysis is performed on the postureinvariant representations. Third, posture-invariant representatives are used to reconstruct mesh shapes to visualize the shape variation.

\subsection{Computing Correspondences}

This section outlines the approach for parameterizing the shapes $S_{1}, S_{2}, \ldots, S_{m}$ over a common domain. Computing correspondences between pairs or a population of shapes is a challenging problem, especially when the shapes deform in a highly non-rigid way. Furthermore, the accuracy of the correspondence information has a large influence on the quality of the resulting shape variations.

In this work, we focus on statistical shape analysis of human shapes. Several methods compute the correspondence information of a set of human models by deforming a template model to each 3D model of a human subject. Allen et al. [4] compute the correspondence information by deforming a template shape using an energy optimization method. This approach requires a sparse set of labeled marker positions and for the subjects of the database to be in a standard posture. $\mathrm{Xi}$ et al. [20] improve this approach by adding a step transforming the template model using a radial basis function based on the known marker positions. Hasler et al. [10] extend Allen et al.'s approach to work for models in varying postures. As before, a set of labeled marker positions is required for this method.

In our experiments, we use two databases: the CAESAR database [1] and the MPI database [10]. The MPI database contains 520 models of 111 different individuals in up to 35 different postures. The models are parameterized using the approach by Hasler et al. [10] and each parameterized model contains 6449 vertices. The markers used for parameterization were placed manually on the scans.

The CAESAR database contains scans of about 5000 different models and all of the subjects were asked to maintain a standard posture. The only posture variation in this database is a slight variation of the arm position. The models are parameterized using the approach by Xi et al. [20] and each parameterized model contains 10002 vertices. The markers used for the parameterization were placed by anthropometric experts prior to scanning the subjects. In our experiments, we use 500 models of the CAESAR database.

As these approaches deform a template model to each scan $S_{i}$, the parameterized models share the same topology and the same spatial resolution in corresponding areas of the models. Hence, in the follow- ing, we do not need to consider problems caused by topological noise or non-isotropic meshes.

\subsection{Posture-Invariant Representation}

We use a method based on the Laplace operator to find posture-invariant representations of the parameterized shapes. The approach proceeds by computing the combinatorial Laplace matrix $L$ of $X_{k}$. The combinatorial Laplace matrix $L$ contains the elements

$$
L_{i j}=\left\{\begin{array}{l}
-1, \text { if } i=j, \\
\frac{1}{\operatorname{deg}\left(v_{i}^{(k)}\right)}, \text { if } v_{j}^{(k)} \in \mathcal{N}_{1}\left(v_{i}^{(k)}\right), \\
0, \text { otherwise, }
\end{array}\right.
$$

where $v_{i}^{(k)}$ denotes the position vector of a vertex of $X_{k}, \mathcal{N}_{1}\left(v_{i}^{(k)}\right)$ denotes the one-ring neighborhood of $v_{i}^{(k)}$, and $\operatorname{deg}\left(v_{i}^{(k)}\right)$ denotes the number of vertices in $\mathcal{N}_{1}\left(v_{i}^{(k)}\right)$.

Since all of the meshes $X_{k}$ are parameterized, the combinatorial Laplace matrices for all of the meshes are the same. This will play a crucial role during the reconstruction step. Note that other isometryinvariant operators, such as the Laplace-Beltrami operator, do not have this property.

Given the Laplace matrix, we can compute the Laplace offsets $\Delta_{i}^{(k)}$ for $X_{k}$ as

$$
\left(\begin{array}{c}
\Delta_{1}^{(k)} \\
\cdots \\
\Delta_{n}^{(k)}
\end{array}\right)=L\left(\begin{array}{c}
v_{1}^{(k)} \\
\cdots \\
v_{n}^{(k)}
\end{array}\right) .
$$

These offsets are not posture-invariant. Hence, we express each offset with respect to the local coordinate system shown in Figure 1 as follows. At each vertex $v_{i}^{(k)}$, we pick an arbitrary but fixed neighbor $v_{j}^{(k)}$ as the first neighbor (we choose the same first neighbor for all of the parameterized meshes). We then compute a local orthonormal coordinate system at $v_{i}^{(k)}$ using the normal vector at $v_{i}^{(k)}$, the normalized projection of the difference vector $v_{j}^{(k)}-v_{i}^{(k)}$ to the tangent plane of $v_{i}^{(k)}$, and the cross product of the previous two vectors. The normal vector is computed as the weighted average of the normal vectors of $v_{i}^{(k)}$, s incident triangles, where the weights are proportional to the triangle areas. We denote the three vectors defining the local orthonormal coordinate system by $f_{1}\left(v_{i}^{(k)}\right), f_{2}\left(v_{i}^{(k)}\right)$, and $f_{3}\left(v_{i}^{(k)}\right)$. Since the local coordinate system is orthonormal, we can express $\Delta_{i}^{(k)}$ in this coordinate system as

$$
\Delta_{i}^{(k)}=\omega_{i}^{1(k)} f_{1}\left(v_{i}^{(k)}\right)+\omega_{i}^{2(k)} f_{2}\left(v_{i}^{(k)}\right)+\omega_{i}^{3(k)} f_{3}\left(v_{i}^{(k)}\right) .
$$

The local coordinates $\omega_{i}^{j(k)}$ are designed to be invariant with respect to rigid transformations of $\mathcal{N}_{1}\left(v_{i}^{(k)}\right)$. In many cases, such as the motion of humans, when the posture of a shape changes, most areas of the shape deform in a locally near-rigid way. 


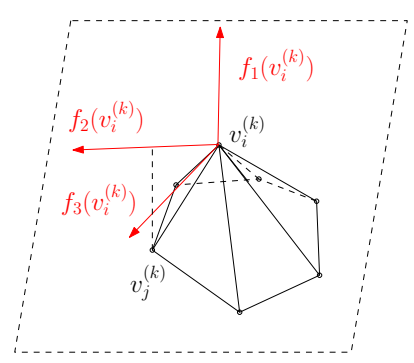

Figure 1: Illustration of the local frame of a vertex $v_{i}^{(k)}$.

Hence, most of the local coordinates $\omega_{i}^{j(k)}$ are not affected by such motions. We therefore propose to perform shape analysis on the local coordinates $\omega_{i}^{j(k)}$ to obtain a posture-invariant shape analysis.

To account for global scaling of the shape, we also store a coefficient $s^{(k)}$ related to the scale of the shape. More specifically, $s^{(k)}$ is computed as the average geodesic distance between any two vertices on $X^{(k)}$. Note that $s^{(k)}$ depends on the resolution of $X^{(k)}$. In our approach, this does not cause problems because all of the parameterized models $X^{(k)}$ have the same resolution. We compute the geodesics using the fast marching technique [21].

\subsection{Shape Analysis}

We perform statistical shape analysis by computing the PCA on the vectors $\omega_{i}^{j(k)}, s^{(k)}$. Compared to performing PCA on the coordinates $v_{i}^{(k)}$ directly, this offers the advantage of being mostly posture-invariant since the local coordinates $\omega_{i}^{j(k)}, s^{(k)}$ only depend on the one-ring neighborhood of the vertex $v_{i}^{(k)}$.

Using PCA, we map each vector of local coordinates $\left[\omega_{1}^{1(k)}, \omega_{1}^{2(k)}, \omega_{1}^{3(k)}, \ldots, \omega_{n}^{1(k)}, \omega_{n}^{2(k)}, \omega_{n}^{3(k)}, s^{(k)}\right]^{T}$ to a vector $W$ of PCA weights. Note that we normalize the PCA space, such that each coordinate axis has unit length. This non-uniform scaling allows us to conduct fair comparisons between different PCA spaces in Section 4. We can visualize the shape variation of the shapes $X_{k}$ by sampling the learned PCA space. It remains to discuss how to compute a mesh based on a given set of ordered local coordinates $\omega_{i}^{j}, s$.

\subsection{Reconstruction}

To visualize the shape variation, we aim to find a mesh $X$ for a given set of ordered local coordinates $\omega_{i}^{j}, s$. Recall that for any mesh

$$
L\left(\begin{array}{c}
v_{1} \\
\cdots \\
v_{n}
\end{array}\right)=\left(\begin{array}{c}
\omega_{1}^{1} f_{1}\left(v_{1}\right)+\omega_{1}^{2} f_{2}\left(v_{1}\right)+\omega_{1}^{3} f_{3}\left(v_{1}\right) \\
\omega_{n}^{1} f_{1}\left(v_{n}\right)+\omega_{n}^{2} f_{2}\left(v_{n}\right)+\omega_{n}^{3} f_{3}\left(v_{n}\right)
\end{array}\right) .
$$

Here, $L$ is known since it is identical for all of the parameterized shapes and $\omega_{i}^{j}$ and $s$ are given. Hence, we aim to find vertex positions $v_{i}$ that satisfy the above equation.
Note that the solution to this problem is not unique since different postures of the same shape may satisfy this equation. We aim to solve the problem using an optimization technique. A good initialization is needed to solve this problem.

We use the following heuristic to solve for the vertex positions $v_{i}$. We choose a mesh $X_{k}$ as template shape and we initialize $v_{i}$ to the vertex positions of the template shape. This allows for the computation of the frames $f_{j}\left(v_{i}\right)$. Equation 1 implies that $v_{i}=$ $\sum_{v_{j} \in \mathcal{N}_{1}\left(v_{i}\right)} \frac{1}{\operatorname{deg}\left(v_{i}\right)} v_{j}-\left(\omega_{i}^{1} f_{1}\left(v_{i}\right)+\omega_{i}^{2} f_{2}\left(v_{i}\right)+\omega_{i}^{3} f_{3}\left(v_{i}\right)\right)$. To improve the initialization, we use this equation to iteratively move $v_{i}$ towards the desired position $d\left(v_{i}\right)=$

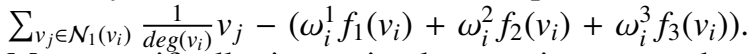
More specifically, in our implementation, we update the vertex positions to $v_{i}^{t+1}=0.5 v_{i}^{t}+0.5 d\left(v_{i}^{t}\right)$, where $t$ is the number of iterations and where $v_{i}^{0}$ is the vertex coordinate of the template shape. The frames $f_{j}\left(v_{i}\right)$ are recomputed in each iteration. We set the number of iterations to 50 .

Once a good initialization is found, we solve Equation 1 by minimizing the energy

$$
\begin{aligned}
E= & \sum_{i}\left(v_{i}-\sum_{v_{j} \in \mathcal{N}_{1}\left(v_{i}\right)} \frac{1}{\operatorname{deg}\left(v_{i}\right)} v_{j}-\right. \\
& \left.\left(\omega_{i}^{1} f_{1}\left(v_{i}\right)+\omega_{i}^{2} f_{2}\left(v_{i}\right)+\omega_{i}^{3} f_{3}\left(v_{i}\right)\right)\right)^{2}
\end{aligned}
$$

using a quasi-Newton approach [22].

For this approach, we compute the gradient of $E$ with respect to the vertex positions $v_{i}$. We use numerical gradients of the local frames for this computation. This is efficient since every local frame only depends on the vertex coordinates of $v_{i}$ and its neighboring vertices. Hence, on average, the numerical derivative of each local frame depends on a small constant number of vertex positions.

The formulation of $E$ and its gradient are simple because we choose the matrix $L$ to be identical for all of the parameterized shapes $X^{(k)}$. If $L$ is chosen differently, for instance as the Laplace-Beltrami matrix, then each mesh may have a different matrix $L$ that depends on the vertex positions $v_{i}$. Hence, in each gradient computation, we would need to compute the derivative of $L$ with respect to $v_{i}$, which would lead to a more complex and less efficient algorithm.

After minimizing $E$, the result has the shape of $X$ in the posture of $X_{k}$. Finally, we scale the result to the desired scale factor $s$.

\section{Experiments}

We analyze the accuracy of the reconstruction step of the algorithm. Furthermore, we perform statistical shape analysis on a population of shapes and compare the results to the results obtained using a standard PCA approach. 


\subsection{Reconstruction Accuracy}

The first experiment demonstrates the accuracy of the reconstruction step. We choose one mesh of the MPI database, denoted by $T$, as template mesh and another mesh of the MPI database, denoted by $X$, to compute the local frames $\omega_{i}^{j}$ and the scale factor $s$. We then compute the reconstruction using the coordinates of $T$ as initial coordinates and $\omega_{i}^{j}$ and $s$ as the desired local coordinates and scale. Figure 2 shows the results. The reconstruction is the body shape of $X$ in the posture of $T$. Note that small geometric details, such as skin folding, are captured in the reconstruction. This shows that the reconstruction algorithm can be used to reposition a body to a standard posture.

Most models of the MPI database were scanned in a standard posture and in multiple other postures. We choose a model in standard posture as template $T$. We evaluate the accuracy of the models by comparing the result of our method to the shape of subject $X$ scanned in standard posture. We denote this model by $M$. The color-coded signed distances (in meters) between the reconstruction and $M$ are shown in Figure 2. Note that most errors on the torso are under $2 \mathrm{~cm}$. Larger errors on the arms and legs are due to the slight posture difference between $T$ and $M$.

Figure 3 shows the back of the model used in the reconstruction shown in the middle of Figure 2. The left side of the figure shows the model $X$ used as input for the reconstruction and the right side of the figure shows the resulting reconstruction. Note that $X$ contains areas with noise, skinny triangles, and selfintersections. While the reconstruction has similar localized problems in the corresponding areas, the overall reconstruction of the shape is correct and areas that were not noisy in $X$ are not affected in the reconstruction. This shows that the method is robust with respect to noise. Furthermore, this shows that the method preserves local features present on the mesh.
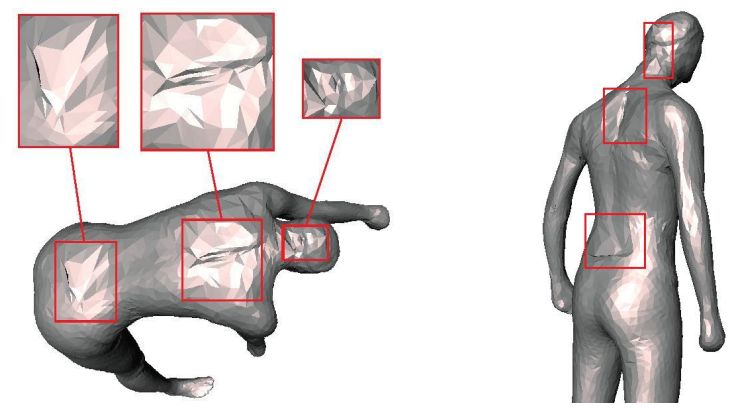

Figure 3: Left: mesh $X$ used as input for reconstruction contains areas with noise, skinny triangles, and self-intersections. Right: reconstruction only contains problems in areas that were noisy in $X$.

\subsection{Influence of Joints}

The second experiment shows the influence of the movement near joints on the local coordinates $\omega_{i}^{j}$. Re- call that $\omega_{i}^{j}$ do not change when the neighborhood of vertex $v_{i}$ is transformed rigidly. The one-ring neighborhood of a vertex near a joint of a human model may not be transformed rigidly when the posture of the model changes. Instead, some of the triangles adjacent to that vertex may stretch. However, if the resolution of the model is high compared to the size of the deformation, we expect that the stretch is distributed over several vertices, and that the change of a specific $\omega_{i}^{j}$ is small.

In the following, we show experimentally that a posture change such as those in the MPI database does not significantly alter the local coordinates in the area around a joint position. Consider the area $A_{\text {blue }}$ around the right elbow shown in blue in the left of Figure 4. For each model of the MPI database, we compute the local coordinates of the vertices in $A_{\text {blue }}$, and we reduce the dimensionality of this data using multidimensional scaling (MDS). This allows us to visualize the data in a way that shows the distances between the data points, as shown in the right of Figure 4. Each point corresponds to the local coordinates in $A_{\text {blue }}$ on one model of the MPI database. The red points show 20 different postures of the same subject. For some of the red points, a screen shot of the corresponding posture is shown and we can observe that the posture of the right elbow varies significantly. Note that the red points form a cluster in the MDS space. This implies that the distances between the local coordinates in $A_{\text {blue }}$ are small for models of the same subject in different postures.

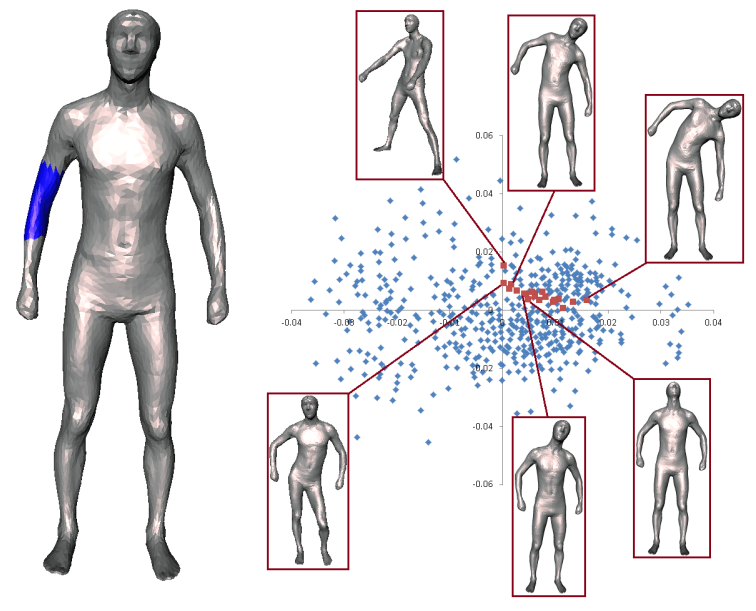

Figure 4: Left: template mesh with highlighted area $A_{\text {blue }}$ around the right elbow. Right: visualization of local coordinates in $A_{\text {blue }}$ over all models of the MPI database. Red points show different postures of the same subject and for some of the red points, a screen shot of the corresponding posture is shown.

\subsection{Shape Analysis}

The third experiment conducts shape analysis using our representation. The most common way to conduct shape analysis on databases containing models 


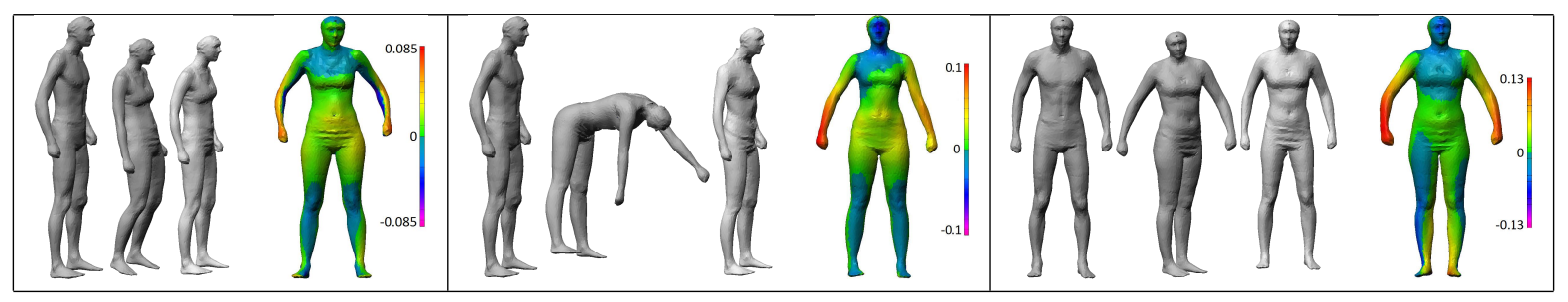

Figure 2: Each sub-figure shows one reconstruction. From left to right: template mesh $T$, mesh $X$ used to compute the local frames $\omega_{i}^{j}$, reconstruction, visualization of error (in meters).

in standard posture is standard PCA. We compare the results obtained using our approach to the results obtained using standard PCA. Recall that our approach computes a PCA space by performing PCA on the representation $\omega_{i}^{j(k)}, s^{(k)}$. For our approach, the meshes do not need to be spatially aligned. Standard PCA computes a PCA space by performing PCA on the vertex coordinates of the meshes after rigidly aligning the meshes using generalized Procrustes analysis [3].

First, we show the result for the CAESAR database. Figure 5 shows the computed PCA spaces. The left side of the figure shows the PCA space obtained using our approach and the right side of the figure shows the PCA space obtained using standard PCA. Note that standard PCA captures arm and body posture movements in the principal components (rotation of shoulder along the first principal component and bending of the elbow and posture change along the second principal component), while our approach only finds shape variations. This shows that our approach contains notably less posture variation than standard PCA even for databases containing subjects in standard posture.

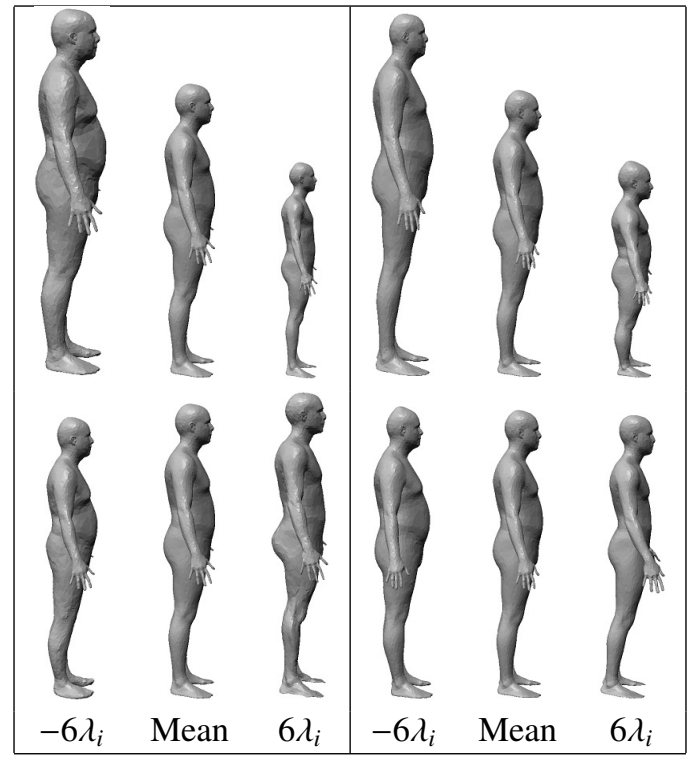

Figure 5: Statistical shape analysis of CAESAR database. The left side shows the results using our approach and the right side shows the results using standard PCA. Each row shows the impact of one principal component (the shapes at distance $6 \lambda_{i}$ from the mean and the mean, where $\lambda_{i}$ is the $i$-th eigenvalue).
For this data set, we evaluate the statistical models generated using our approach and using standard PCA with the help of the three error measures suggested by Styner et al. [23]: compactness, generalization, and specificity. A compact model has little variance and it requires few principal components to describe the model, a general model has the ability to describe instances that are not in the training set, and a specific model only generates shapes that are similar to the ones in the training set.

More specifically, compactness is defined as $C(k)=$ $\sum_{i=1}^{k} \lambda_{i} / \sum_{i=1}^{m} \lambda_{i}$, where $k$ is the number of principal components that are kept, $m$ is the number of models in the training set, and where $\lambda_{i}$ is the $i$-th eigenvalue. The compactness error is computed as $\sigma_{C(k)}=$ $\sqrt{2 / m} C(k)$. To measure generalization $G(k)$, we compute a statistical model using the first $k$ eigenvectors with all but one of the corresponded scans of the training set and fit the model to the excluded scan. Generalization measures the accuracy of the description of the excluded scan and is computed as the distance from the original model to its projection onto PCA space, averaged over the complete set of trials. The standard error is computed using the standard deviation $\sigma$ of errors of the complete set of trials as $\sigma_{G(k)}=\sigma / \sqrt{m-1}$. Specificity $S(k)$ is defined as the average distance of $S_{\text {rand }}$ to its nearest neighbor in the training set, where $S_{\text {rand }}$ is a shape computed using the statistical model from a uniformly distributed random sample in PCA shape space. The standard error is computed using the standard deviation $\sigma$ of the distances as $\sigma_{S(k)}=\sigma / \sqrt{m}$.

Figure 6 gives the error measures. Note that our method leads to lower errors for all three evaluation measures. This is to be expected because our method represents the statistical model in a posture-invariant way while standard PCA encodes posture variations in the statistical model.

Second, we show the result for the MPI database. Figure 7 shows the computed PCA spaces. The left side of the figure shows the PCA space obtained using our approach and the right side of the figure shows the PCA space obtained using standard PCA. When analyzing the shape variations found using our approach, we can see that the principal components capture shape differences rather than posture differences 


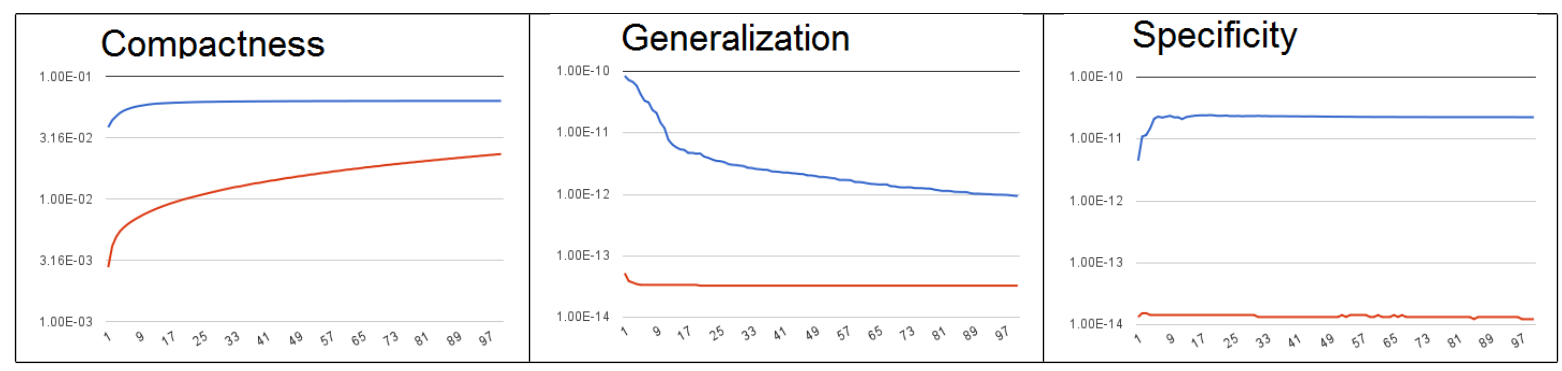

Figure 6: Error evaluation. The result using standard PCA is shown in blue and the result using our method is shown in red. The x-axis shows the number of principal components and the y-axis shows values of the error measures.

and that visually realistic body shapes are obtained. This shows that our approach is suitable for analyzing the shape changes of databases in varying posture.

However, it is well-known that standard PCA is not suitable for analyzing the shape changes of databases in varying postures. This can be seen when analyzing the shape variations found using standard PCA. We can see that the standard PCA approach mostly captures posture changes. Furthermore, the standard PCA approach leads to unrealistic body shapes, as can be seen when looking at the arms in the first row of the right side of the figure.

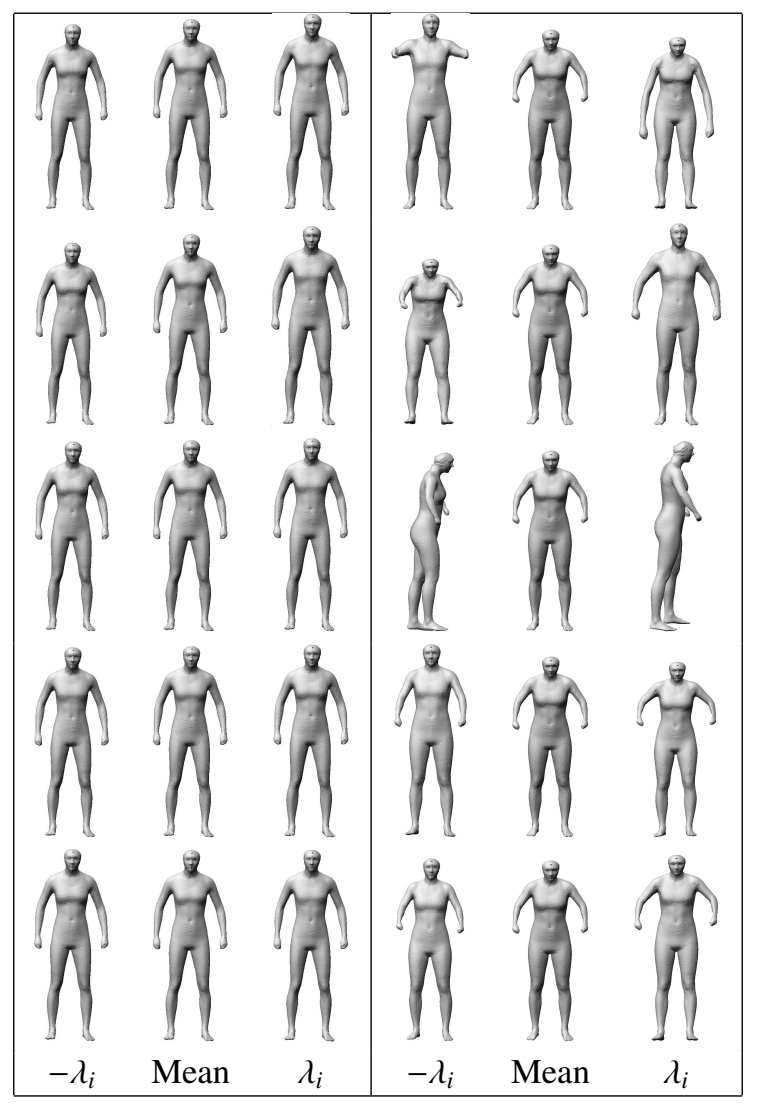

Figure 7: Statistical shape analysis of MPI database. The left side shows the results using our approach and the right side shows the results using standard PCA. Each row shows the impact of one principal component (the shapes at distance $\lambda_{i}$ from the mean and the mean, where $\lambda_{i}$ is the $i$-th eigenvalue).

\subsection{Application to Estimating Body Shape of Dressed Subject}

Statistical shape models are often useful for synthesizing new shapes from partial information. As an application of our learned shape space, we estimate the body shape from a scan of a dressed subject. To obtain a parameterization that is consistent with our training data, we first fit a template model to the scan shown on the left of Figure 8 using an approach similar to the one used by Hasler et al. [10] to register a database of scans. The result is shown in the middle of Figure 8, and we can see that the surface includes the shape of the clothing. To estimate the body shape of the subject, we compute the local coordinates of the deformed template, project the result onto to the PCA space learned using the MPI database (using the first 15 principal components), and use the point in PCA space to reconstruct the body shape. The template fitted to the scan is used as initialization when reconstructing the body shape. The right of Figure 8 shows the result. Table 1 gives an evaluation of the accuracy of the model using a set of the measurements. All of the measurements except the waist girth are within $3 \mathrm{~cm}$ of the true measurements, which is fairly accurate. The waist girth of our estimate is higher than the true waist girth because the subject was scanned while wearing a loose sweater. The loose clothing leads to a surface that is far from the true body surface in the area of the waist. Hence, the scan observation could be explained by human body shapes with different waist girths. Our estimate is a solution that is visually consistent with the scan. This demonstrates that our learned shape space can be applied to synthesize new shapes based on partial observations.

\begin{tabular}{|l|r|r|r|r|}
\hline & Height & Arm Length & Leg Length & Waist Girth \\
\hline Ground truth & 182 & 57 & 79 & 74 \\
\hline Estimate & 185 & 60 & 77 & 85 \\
\hline
\end{tabular}

Table 1: Biometric measurements (in cm). The first row shows the ground truth measurements and the second row shows the corresponding measurements on the estimated body shape. 


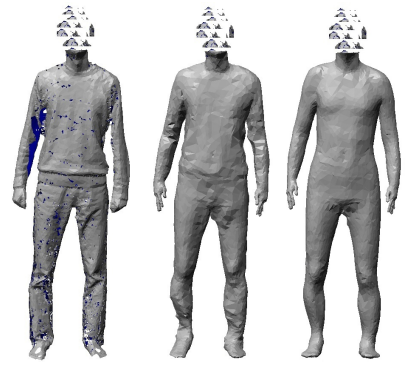

Figure 8: Left: scan data from [24]. Middle: template model fitted to scan. Right: estimated body shape.

\section{Conclusion}

We proposed an approach to perform statistical shape analysis in a posture-invariant way. This approach was shown to allow for the analysis and visualization of shape variations in a database of human models in varying postures. This is useful for design applications, where one is interested in visualizing the shape variations of a population in varying postures while ignoring posture variations.

The reconstruction of a triangle mesh based on a set of ordered local coordinates is currently solved using a heuristic energy optimization scheme. An interesting question for future work is to find more robust ways to solve this problem.

\section{Acknowledgments}

We thank Nils Hasler for providing us with the MPI database and the scanned clothed subject. We thank Jonathan Boisvert and Alan Brunton for helpful discussions on the topic, and the anonymous reviewers for insightful comments. This work has partially been funded by the Cluster of Excellence Multimodal Computing and Interaction within the Excellence Initiative of the German Federal Government.

\section{References}

[1] K. Robinette, H. Daanen, E. Paquet, The CAESAR project: A 3-D surface anthropometry survey, in: 3-D Digital Imaging and Modeling, 1999, pp. 180-186.

[2] O. van Kaick, H. Zhang, G. Hamarneh, D. Cohen-Or, A survey on shape correspondence, in: Eurographics State-of-theart Report, 2010.

[3] I. Dryden, K. Mardia, Statistical Shape Analysis, Wiley, 2002.

[4] B. Allen, B. Curless, Z. Popović, The space of human body shapes: reconstruction and parameterization from range scans, ACM Transactions on Graphics 22 (3) (2003) 587-594, proceedings of SIGGRAPH.

[5] P. T. Fletcher, C. Lu, S. M. Pizer, S. Joshi, Principal geodesic analysis for the study of nonlinear statistics of shape, IEEE Transactions on Medical Imaging 23 (2004) 995-1005.

[6] D. Nain, M. A. Styner, M. Niethammer, J. J. Levitt, M. E. Shenton, G. Gerig, A. F. Bobick, A. Tannenbaum, Statistical shape analysis of brain structures using spherical wavelets., in: IEEE International Symposium on Biomedical Imaging, 2007, pp. 209-212.
[7] Y. Wang, J. Zhang, T. F. Chan, A. W. Toga, P. M. Thompson, Multivariate tensor-based morphometry on surfaces: Application to mapping ventricular changes in hiv/aids., in: IEEE International Symposium on Biomedical Imaging, 2009, pp. 129-132.

[8] M. A. O. Vasilescu, D. Terzopoulos, Multilinear (tensor) ica and dimensionality reduction, in: Proceedings of the 7th international conference on Independent component analysis and signal separation, 2007, pp. 818-826.

[9] D. Anguelov, P. Srinivasan, D. Koller, S. Thrun, J. Rodgers, J. Davis, Scape: shape completion and animation of people, ACM TOG 24 (3) (2005) 408-416, proceedings of SIGGRAPH.

[10] N. Hasler, C. Stoll, M. Sunkel, B. Rosenhahn, H.-P. Seidel, A statistical model of human pose and body shape, Computer Graphics Forum (Special Issue of Eurographics 2008) 2 (28).

[11] T. B. Moeslund, A. Hilton, V. Krueger, A survey of advances in vision-based human motion capture and analysis, Computer Vision and Image Understanding 104 (2-3) (2006) 90126, special Issue on Modeling People: Vision-based understanding of a persons shape, appearance, movement and behaviour.

[12] A. Nealen, T. Igarashi, O. Sorkine, M. Alexa, Laplacian mesh optimization, in: International Conference on Computer graphics and Interactive Techniques in Australasia and Southeast Asia, 2006, pp. 381-389.

[13] O. Sorkine, D. Cohen-Or, Y. Lipman, M. Alexa, C. Rössl, H.-P. Seidel, Laplacian surface editing, in: Symposium on Geometry Processing, 2004, pp. 175-184.

[14] I.-C. Yeh, C.-H. Lin, O. Sorkine, T.-Y. Lee, Template-based $3 \mathrm{~d}$ model fitting using dual-domain relaxation, IEEE Transactions on Visualization and Computer Graphics 99.

[15] B. Vallet, B. Lévy, Spectral geometry processing with manifold harmonics, Computer Graphics Forum (Proceedings Eurographics).

[16] M. Reuter, M. Niethammer, F.-E. E. Wolter, S. Bouix, M. Shenton, Global medical shape analysis using the volumetric laplace spectrum., Lecture Notes in Computer Science 2007 (2007) 417-426.

[17] H.-Y. Wu, H. Zha, T. Luo, X. Wang, S. Ma, Global and local isometry-invariant descriptor for $3 \mathrm{~d}$ shape comparison and partial matching, in: IEEE Conference on Computer Vision and Pattern Recognition, 2010, pp. 438-445.

[18] I. Baran, D. Vlasic, E. Grinspun, J. Popović, Semantic deformation transfer, ACM Transactions on Graphics 28 (3) (2009) 36:1-6, proceedings of SIGGRAPH.

[19] Y. Yu, K. Zhou, D. Xu, X. Shi, H. Bao, B. Guo, H.-Y. Shum, Mesh editing with poisson-based gradient field manipulation, ACM Transactions on Graphics 23 (3) (2004) 644-651, proceedings of SIGGRAPH.

[20] P. Xi, W.-S. Lee, C. Shu, Analysis of segmented human body scans, in: Graphics Interface, 2007.

[21] R. Kimmel, J. Sethian, Computing geodesic paths on manifolds, Proceedings of the National Academy of Sciences 95 (1998) 8431-8435.

[22] D. C. Liu, J. Nocedal, On the limited memory method for large scale optimization, Mathematical Programming 45 (1989) 503-528.

[23] M. Styner, K. T. Rajamani, L.-P. Nolte, G. Zsemlye, G. Székely, C. J. Taylor, R. H. Davies, Evaluation of 3d correspondence methods for model building, in: Information Processing in Medical Imaging, 2003, pp. 63-75.

[24] N. Hasler, C. Stoll, B. Rosenhahn, T. Thormählen, H.-P. Seidel, Estimating body shape of dressed humans, Computers and Graphics 33 (3) (2009) 211-216, proceedings of Shape Modeling International. 